\title{
Classification of Age from Facial Features of Humans
}

\author{
Poonam Shirode ${ }^{1}$, S. M. Handore ${ }^{2}$ \\ ${ }^{1,2}$ Department of E\&TC, K.J's Educational Institute's TCOER, Pune, Maharashtra, India
}

\begin{abstract}
This writing presents a new way for classification of human age based on $k$-NN classifier. The hypothesis has been implemented to distribute input images into one of four age groups i.e kid, young, adult and senior adult. For implementation, first primitive features of face supported on craniofacial development is found. For subordinate features skin texture and wrinkle analysis is found. Images from FGNET database are used to differentiate babies from other groups based on geometrical ratios. The other three groups are classified by LBP for skin texture and Gabor for wrinkles analysis. Finally, age group are categorized by using $k$-NN classifier.
\end{abstract}

Keywords: Age classification, Geometric Feature, LBP, Gabor, k-NN classifier.

\section{Introduction}

As one of the main human facial characteristic, oldening plays a more intricate role than other element such as human selfhood, expression, gender and race. The progress of human aging is ungovernable, with many interior and exterior ascendency element such as one's health condition, lifecycle and extreme weather conditions. Besides, since age is a temporal property of populate, it's difficult to gather the same person's face copy across ages. It's also irksome and tiresome to tag the precise or approximate ages of collected faces. Due to these difficulties, researches on human age are not as much as that on other facial characteristic.

However, researches on age progression and estimation have large influential in many applications, e.g. homeland safety, parental control, age based Human-Computer interaction, passports verification and finding missing individuals, and in particular face recovery over internet or large scale face copy databank that is our targeted application area. In literature, dissimilar aspects of age progression are exploration, hold construction complex design to predicate or simulate one's facial look in futurition[1], esteem or rank the age of a given face copy [2][4][5][6], age progression modeling to alleviate performance drop in face recognition [3][7].

\section{Literature Survey}

Some former work has been reported on different aspects of age progression and estimation. Kwon and Lobo design an age classification system that concentrate on both shape and wrinkles of the human face to group input images into only one of the three era groups such as babies, young adults and senior adults [8]. Lanitis et al. [9] design a tetragonal oldening function that maps the Active appearance model (AAM) form of a face image to an age.

The creator also obtains separate classifiers for age calculation supported on AAM characteristics in their later work [10]. With AAM based face encoding, Geng et al. [11], [12] handled the age estimation problem by introducing an aging pattern subspace (AGES), which is a subspace resemblance of arrangement of individual oldening face images.

Yang and Ai [13] used Real AdaBoost algorithm to exercise a classifier by composing a consequence of Local Binary Pattern (LBP) features as a representation of face texture. Age is categorised into only three periods: child, adult and oldness. Guodong Guo et al introduced the age manifold literature plan for extracting face aging charecteristics and have proposed a locally regulated robust regressor for learning and prediction of human ages [14]. Gao and Ai used Gabor features as face representation and the Linear Discriminant Analysis (LDA) to construct the age classifier that classifies human faces as baby, child, adult, or elder people. Images in the training set are labeled without the age information [15].

More recently, Yuille, Hallinan, and Cohen [16] usage deformable patter to find out characteristic of faces, in appropriate, eyes and lips. External energy functions of valley, peak, edge, and image intenseness are determined. These exterior images are calculate from the source intensity image. The interior energy consist of the patter parameters interact dynamically with the exterior energy.

The most appropriate appear when the complete energy equation,consist of interior and exterior energy, is minimized.Edwards et al. [17] describe how a shape model and an intensity model can be combined in order to produce a combined shape-intensity face model capable of modelling effectively both shape and intensity variation.

The effective texture descriptor, Local Binary Patterns (LBP) [18], has been used for appearance feature extraction in an automatic age estimation system [19]. It achieves $80 \%$ accuracy on the FERET database [20] with nearest neighbor classification, and $80 \%-90 \%$ on the FERET and PIE databases with AdaBoost [21]. The Gabor feature [22] has also been tried on the age estimation task [23], which has been demonstrated to be more effective than LBP 


\section{Overview of Proposed Method}

The proposed method for age classification includes feature extraction, a feature fusion and age classification as shown in Figure.1. In feature extraction, skin and wrinkle features are extracted and combined together in feature fusion. Then input images are classified into different age groups by k-NN classifier.

\section{Paper Organization}

The remainder of the paper is organized as follows. In Section II, we present a brief overview of the relevant bibliography. In Section III, we briefly describe how the proposed method models work. In Section IV, we present the database used in our experiments, in Section V, we present the results obtained. Finally, in Section VI, we present our conclusion

\section{Proposed Work}

The proposed age classification system is concisely sketch in this paragraph. The system process is sketch into stages as characteristic extraction and age classification as illustrated in Figure 1.Initially an image is acquired from databank and for this work FGNET databank is necessary. The input image is preprocessed and used for face detection and then for feature extraction. Geometric features are used for aging details in craniofacial growth. Geometric characteristic are obtained using placing of eyes, nose and mouth. Geometric ratios are calculated on find out face image. Using these ratios the threshold is resolved which is used to differentiate child from young, adult, senior adult. Both geometrical and wrinkle characteristic are employed in the system for age classification.

To differentiate young, middle aged and old, we extract wrinkle feature. Division of lifetime age groups is done in age classification .In age classification phase; geometric features are used to distinguish whether a facial conception in children. If it is not, the provincial base 2 patterns are used for texture analysis and Gabor for winkle discovery. As the moving sift of each facial effigy is different. Thus, the preprocessing of histogram equaling action is performed on all facial images on which the experimentation to be carried out, so that the ramble of gray level of all the likeness are mapped [0,255].

\subsection{Preprocessing}

In preprocessing the RGB image is reborn to gray first. Noise is removed from the gray image using median filter .Median filtering is a nonlinear procedure noteful in reducing impulsive or salt-and-pepper noise. It is also useful in sustain edges in an copy while reducing random noise. In a median filter, a window move along the copy and the median intenseness worth of the pixels within the window becomes the product intensity of the pixel being processed. Like lowpass filtering, median filtering smoothies the image and is thus useful in reducing noise. Unlike low-pass filtering, median filtering can sustain discontinuities in a step function and can smooth a few pixels whose values vary way from their surroundings without affecting the other pixels. The Canny edge detector is an edge detection speculator that uses a several-stage algorithm to find out a wide range of sharpness in images. Among the margin discovery methods improved so widely, canny edge discovery algorithmic rule is one of the most precisely determine methods that contribute useful and trustworthy discovery. Histogram equalization is a method in image processing of contrast arrangement using the copy's histogram. This method usually extend the global contrast of many images, chiefly when the usable data of the copy is represented by close contrast values. Image segmentation is the process of division a digital image into manifold segments. The goal of division is to simplify and/or change the portraiture of an image into something that is more meaningful and easier to analyze.

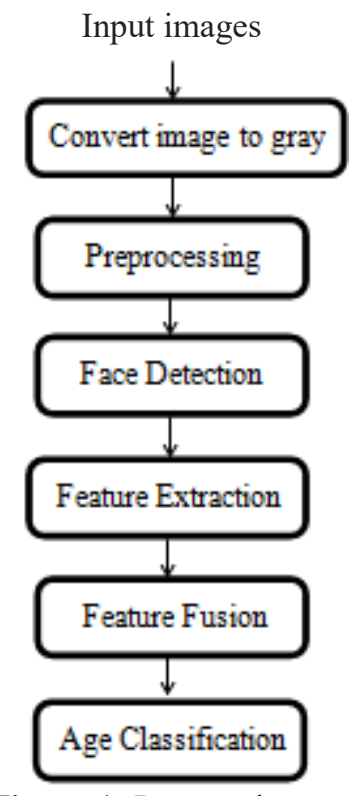

Figure 1: Proposed system

\subsection{Face Detection}

To analyze the facial information we use Viola-Jones object detection framework. The Viola-Jones object detection framework is the first object detection framework to provide competitive object detection rates in real time proposed in 2001 by Paul Viola and Michael Jones. This algorithm processes the data in real time like 2 frames per second.

The algorithm has four stages

Haar Feature Selection: All human face share some common properties like eye region is darker than upper cheeks, the nose bridge region is brighter than the eyes. Composition of properties forming matchable facial features like location and size of eyes, mouth, bridge of nose, value of oriented gradients of pixel intensities. Viola \& Jones used two rectangle features having value as

Value $=\Sigma$ (pixels in black area) $-\Sigma$ (pixels in white area) Creating an Integral Image: A summed area table is a data structure and algorithm for quickly and efficiently generating the sum of values in a rectangular subset of a grid. In the image processing domain, it is also known as an integral image. Adaboost Training: This algorithm is used to boost the application performance. This algorithm is sensitive of 


\section{International Journal of Science and Research (IJSR) \\ ISSN (Online): 2319-7064}

Index Copernicus Value (2013): 6.14 | Impact Factor (2014): 5.611

noisy data. Cascading Classifiers: All the features are grouped into several stages where each stage has certain number of features having strong classifiers generated by the learning process. The job of each stage is to determine whether a given sub-window is definitely not a face or may be a face. A given sub-window is immediately discarded as not a face if it fails in any of the stages.

\subsection{Feature Extraction}

In classification system the main issue is to find a set of features on which categorization of ages can be done as many facial features change with increasing age. For features extraction we are using geometrical features, LBP(Local Binary Pattern) for skin feature extraction and gabor features for wrinkle detection. By using these feature extraction techniques different features of the human face are identified such as finding eyes, nose, mouth, chin, skin tone, wrinkles. Further these feature are used to perform the different classification operation like geometrical classification and LBP classification to get the exact age of the person.

\subsubsection{Geometrical Features}

Geometrical feature are used for aging detail in craniofacial growth. It is used to find baby from other age groups by obtaining different ratios between eyes, nose, mouth, chin and virtual top of head.

Here we evaluate the six ratios to find out the age of the person,

Ratiol is calculated by finding the distance between left eye and right eye and distance between eye and nose.

Ratio2 is calculated by finding the ratio of distance between left eye and right eye and distance between eye and mouth.

Ratio3 is calculated by finding theratio of distance between left eye and right eye and distance between eye and chin.

Ratio4 is calculated by finding theratio of distance between eye and nose and distance between eye and mouth.

Ratio5 is calculated by finding theratio of distance between eye and mouth and distance between eye and chin.

Ratio6 is calculated by finding theratio of distance between eye and chin and distance between virtual top of head and chin.

These ratios need to more analyze to improve the correctness in calculating the person age as the image get rotated the ratio causes the error in the calculation. Hence we need to calculate the multiple ratios to get the clear result.

\subsubsection{Local Binary Pattern}

In texture analysis, skin and wrinkle features are extracted to find out young people and aged people. Local binary pattern (LBP) is used for skin aging analysis which is capable of extracting detailed texture of skin. As a person getting older, facial spots increases which are used to find young age group from other age groups. The overall tone of the facial skin becomes non uniform as it gets damaged by the exposure to ultra violate rays from the sun. The aging of skin is mainly affected by the exposure in sunlight and the most affected parts are cheeks and bridge of nose. Thus, LBP is used to detect the edges, lines and spot areas. The LBP operator assigns a code to each pixel comparing to its neighboring pixels. LBP code is expressed as in Eq. (1)

$$
L B P_{P, R}=\sum_{p=0}^{p-1} s\left(g_{p}-g_{c}\right) 2^{p}, \text { where } s(x)=\left\{\begin{array}{l}
1, x \geq 0 \\
0, x<0
\end{array}(1)\right.
$$

Where $\mathrm{P}$ used for the number of neighboring pixels, $\mathrm{R}$ used for the distance from the center to the neighboring pixels, $g_{c}$ Corresponds to the gray value of the center pixel, $\mathrm{s}$ is the threshold function of $\mathrm{x}$, and $g_{p}(p=1, \ldots, P-1)$ corresponds to the gray values of the $\mathrm{p}$ equally spaced pixels on the circle of radius $\mathrm{R}(\mathrm{R}>0)$ that forms a circularly symmetric neighbor set.

\subsubsection{Gabor Filter}

Wrinkles are the indication of aging. Thus it used to indicate aged people. Aged people usually have wrinkles skin on forehead, corners of eyes and cheeks. The facial wrinkles gives important information related to age estimation. Facial wrinkles depend upon the direction of muscles and the amount by which they are used. A set of Gabor filter is used to extract wrinkles from face. In each wrinkles area, the mean and variance of the magnitude response of Gabor filter is used. As quantity of wrinkles are represented by the mean and variance of the magnitude. The geometric features are defined in Eq. (2).

$$
g(x, y)=\left(\frac{1}{2 \pi \sigma_{x} \sigma_{y}}\right) \exp \left[-\frac{1}{2}\left(\frac{x^{2}}{\sigma_{x}^{2}}+\frac{y^{2}}{\sigma_{y}^{2}}\right)+2 \pi j W x\right]
$$

Where, $\sigma_{\mathrm{x}}$ and $\sigma_{\mathrm{y}}$ are the standard deviations of the $\mathrm{x}$ - and $\mathrm{y}-$ axes, respectively, and $\mathrm{W}$ is the radial frequency of the sinusoid. Figure . 2 shows the 24 Gabor filters that have the scale number of $S=4$, the orientation number of $K=6$.

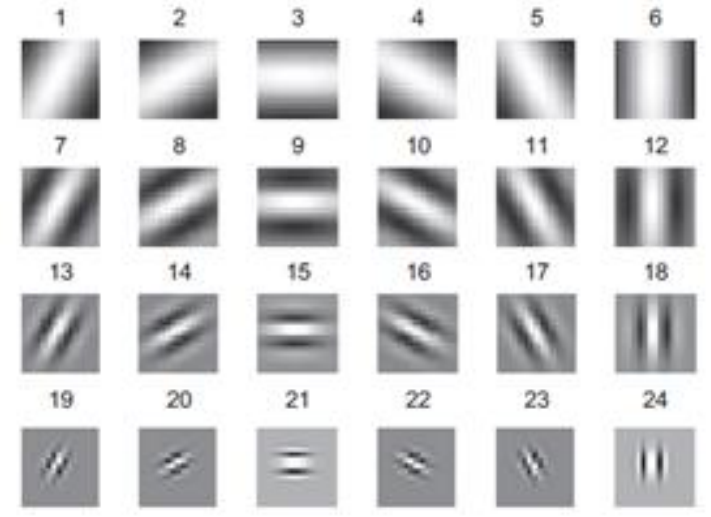

Figure 2: Gabor filter with 4 scale and 6 orientation

\subsection{Feature Fusion}

Extracted features from geometrical features and texture features are fused together to find out various age groups. These feature fusion create the single row having the $n$ number of columns. These data is further used by the different classifiers to determine the age of the person by comparing these data with the training dataset.

\subsection{Age Classification}

To classify the age of the person we use k-NNclassifier for classification mechanism.k-NN stands for "k-nearest neighbor algorithm", it is one of the simplest but extensively using machine learning algorithmic. An object is categorized by the "distance" from its adjacent neighbors, with the object

\section{Volume 4 Issue 11, November 2015}




\section{International Journal of Science and Research (IJSR) \\ ISSN (Online): 2319-7064}

Index Copernicus Value (2013): 6.14 | Impact Factor (2014): 5.611

being assigned to the class most common among its $\mathrm{k}$ distance-nearest neighbors.

Euclidean distance is to calculate distance between two vector positions in the multidimensional space. The Euclidean distance between $\mathrm{X}=(\mathrm{x} 1, \mathrm{x} 2, \mathrm{x} 3, \ldots \mathrm{xn})$ and $\mathrm{Y}$ $=(\mathrm{y} 1, \mathrm{y} 2, \mathrm{y} 3, \ldots \mathrm{yn})$ is defined as in Eq. (3)

$$
D(X, Y)=\sqrt{\sum_{i=1}^{n}\left(x_{i}-y_{i}\right)^{2}}
$$

$\mathrm{k}-\mathrm{NN}$ classifier is used for human age classification. k-NN starts computing the sample data generated by the training set by using the feature extracted by different methods, using the appropriate nearest neighbor search algorithm k-NN group the feature data with specific extracted feature and compute the data for $\mathrm{k}$ times to get the exact result. The compact function reduces the size of most classification models by removing the training data properties and other properties that are not required to predict the new observation as k-NN classification models require all of the training data to predict the result.

$\mathrm{k}-\mathrm{NN}$ works on the majority voting among the data records in the neighborhood is used to decide the classification for the various points with or without consideration of distance based weighting. To apply k-NN we need to choose a proper value for $\mathrm{k}$, because the success of the $\mathrm{kNN}$ is very much dependent on the value of $\mathrm{k}$. we can select the value of $\mathrm{k}$ by trying the multiple value of $\mathrm{k}$ to choose the best performance for the value.

\section{Database}

The age of subjects in FG-NET ranges from 0-69 years, over $50 \%$ of the subjects in FG-NET are between the ages 0 and 13. FG-NET[24] consists of more than 1,000 images of 82 individuals. In our work we used 109 images .The average number of images per individual is 12 . This sample data provided into the database is then used for the training the system, which is more useful in the further analysis.

We use this training data for different feature analysis using LBP, Gabor amd geometric ratios. Analysis of the given training set on the basis of LBP, geometric and Gabor feature extraction is given in the below Figure 3 .

\begin{tabular}{|c|c|c|c|c|}
\hline & Intensity & LBP & Geometric & Gabor \\
\hline Baby (1.5) & 99.2000 & 79.8617 & 25 & 100.8643 \\
\hline Baby $(6-10)$ & 94.4000 & 829675 & 30 & 109.7668 \\
\hline Child(11-15) & 97.9000 & 81.1465 & 25 & 83.3077 \\
\hline Child(16-20) & 100.3000 & 89.5727 & 29.4000 & 97.0911 \\
\hline Adult(21-30) & 958000 & 83.4306 & 25 & 180.7295 \\
\hline Adult( $(31-40)$ & 99.2000 & 20.2621 & 25 & 1259742 \\
\hline $\operatorname{Old}(41-50)$ & 89.2000 & 83.7794 & 30 & 104.0567 \\
\hline Old(50 Above) & 8s: 8000 & 759767 & 759767 & 122.4759 \\
\hline Total Precision & 190.4500 & 164.2493 & 68.3442 & 246.0665 \\
\hline
\end{tabular}

Figure 3: Age classification precision - Training set

\section{Result}
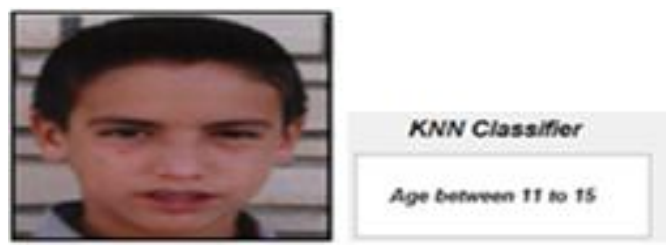

\section{Conclusions}

An age classification system has been proposed in this paper. Age classification system consist of feature extraction . In this paper feature extraction method for skin and wrinkles texture detection has been proposed. Gabor filter method is used for wrinkle detection and LBP are used for skin texture detection. The experimental results show that the proposed system provides better results than the previous methods. To find out the result use the $\mathrm{kNN}$ algorithm. Classifier use the training database to calculate the age group of the person in the image for the given proposed system. FGNet aging database is used to get the sample data for the comparison which work as a training dataset into the age detection classifier algorithms. FGNet aging database does not have the enough information related to aging based on the wrinkle features analysis, so the wrinkle feature information provided into the database need to rich so that this database can be more efficiently used to identify the persons age. The future scope of this work is that we can find expression, light and gender from facial images.

\section{References}

[1] Sung EunChoi , YounJooLee, SungJooLee, KangRyoungPark, JaihieKim, "Age estimation using a hierarchical classifier based on global and local facial features" Elsevier Journal Proc. Pattern Recognition 44 (2011) 1262-1281

[2] Kwon, Y. H. and da Vitoria Lobo, N., "Age classification from facial images," Proc. IEEE Conf. on Computer Vision and Pattern Recognition, Seattle, Washington, U. S. A., pp. $762-767$ (1994).

[3] AgeProgression,http://www.forensicartist.com/agepro.ht $\mathrm{ml}$.

[4] N. Ramanathan and R. Chellappa, "Face Verification Across Age Progression," IEEE Trans. on Image Processing, vol. 15, no. 11, pp. 3349-3361, 2006.

[5] G. Guo, Y. Fu, C. Dyer, and T. S. Huang, "Image-Based Human Age Estimation by Manifold Learning and Locally Adjusted Robust Regression," IEEE Trans. on Image Processing, vol. 17, no. 7, pp. 1178-1188, Jul. 2008.T. R. Alley, Social and Applied Aspects of Perceiving Faces, Lawrence Erlbaum Associates, Hillsdale, NJ, 1988]

[6] Y. Fu, Y. Xu, and T. S. Huang, "Estimating Human Ages by Manifold Analysis of Face Pictures and Regression on Aging Features," Proc. IEEE Conf. on Multimedia and Expo, pp. 1383-1386, 2007.

[7] N. Ramanathan and R. Chellappa, "Modeling Age Progression in Young Faces," Proc. IEEE Conf. Computer Vision and Pattern Recognition, pp. 387-394, 2006.

[8] Kwon, Y. H. and Lobo, N. D. V. " Age Classification

\section{Volume 4 Issue 11, November 2015}




\section{International Journal of Science and Research (IJSR) \\ ISSN (Online): 2319-7064}

Index Copernicus Value (2013): 6.14 | Impact Factor (2014): 5.611

from Facial Images," In Proc. of IEEE Conference on Computer Vision and Pattern Recognition, pp.762-767, 1999.

[9] A. Lanitis, C. Taylor, and T. Cootes, "Toward Automatic Simulation of Aging Effects on Face Images," IEEE Transactions on Pattern Analysis and Machine Intelligence, vol. 24, no. 4, pp. 442-455, 2002.

[10]A. Lanitis, C. Draganova, and C. Christodoulou, "Comparing Different Classifiers for Automatic Age Estimation," IEEE Transactions on Systems, Man, and Cybernetics, vol. 34, no. 1, pp. 621-628, 2004.

[11]X. Geng, Z. Zhou, and K. Smith-Miles, "Automatic Age Estimation Based on Facial Aging Patterns," IEEE Transactions on Pattern Analysis and Machine Intelligence, vol. 29, no. 12, pp. 2234-2240, 2007.

[12]X. Geng, Z.-H. Zhou, Y. Zhang, G. Li, and H. Dai, "Learning from Facial Aging Patterns for Automatic Age Estimation," in Proc. of ACM Multimedia, 2006, pp. 307-316.

[13] Yang, Z. G. and Ai, H. Z. "Demographic Classification with Local Binary Patterns," In Proc. IEEE Conference on Computer Vision and Pattern Recognition, pp. 464 473, 2007.

[14] G. Guo, G. Mu, Y. Fu, and T. Huang, "Human Age Estimation using Bioinspired Features," in Proc. of IEEE Conference on Computer Vision and Pattern Recognition, pp. 112-119, 2009.

[15] Gao, F. and Ai, H. "Face Age Classification on Consumer Images With Gabor Feature and Fuzzy LDA Method," In Proc. of the Third International Conference on Advances in Biometrics, ICB '09,Berlin,Heidelberg. Springer-Verlag, pp.

[16] A. L. Yuille, P.W. Hallinan, and D. S. Cohen, Feature extraction from faces using deformable templates IJCV 8, No. 2 (1992), 99-111.

[17] G. J. Edwards, A. Lanitis, C. J. Taylor, and T. F. Cootes, "Statistical face models: Improving specificity," Image Vis. Comput., vol. 16, no. 3, pp.203-211, 1998.

[18] T. Ahonen, A. Hadid, and M. Pietikainen, "Face Description with Local Binary Patterns: Application to Face Recognition," IEEE Trans. Pattern Anal. Mach. Intell., vol. 28, no. 12, pp. 2037-2041, 2006.

[19]A. G"unay and V. Nabiyev, "Automatic Age Classification with LBP," Proc. International Symposium on Computer and Information Sciences, 2008.

[20] P. J. Phillips, H. Moon, S. A. Rizvi, P. J. Rauss, "The FERET Evaluation Methodology for Face Recognition Algorithms," IEEE Trans. Pattern Anal. Mach. Intell., vol. 22, no. 10, pp. 1090-1104,2000.

[21]Z. Yang and H. Ai, "Demographic Classification with Local Binary Patterns," Proc. International Conference on Biometrics, Lecture Notes In Computer Science, vol. 4642, pp. 464-473, 2007.

[22]C. Liu and H. Wechsler, "Gabor Feature Based Classification Using the Enhanced Fisher Linear Discriminant Model for Face Recognition," IEEE Trans. Image Processing, vol. 11, no. 4, pp.467-476, 2002.

[23]F. Gao and H. Ai, "Face Age Classification on Consumer Images with Gabor Feature and Fuzzy LDA Method," Proc. International Conference on Advances in Biometrics, Lecture Notes In Computer Science, vol. 5558,pp.132-141,2009.
[24] http://www.fgnet.rsunit.com/

\section{Author Profile}

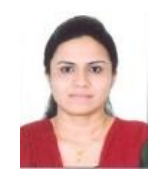

Poonam Ulhas Shirode received the B.E degree in Electronics andTelecommunication Engineering from Pune University, Maharashtra State, India in 2011. Presently a Masters Student of Digital Systems in Pune University, Maharashtra, India .Works as a Lecturer in Zeal Polytechnic, Narhe, Pune, Maharshtra, India. 